\title{
A Non-Cryogenic Method for the Preparation of 2-(Indolyl) Borates, Silanes and Silanols.
}

\author{
Enrique Vazquez,* Ian W. Davies, and Joseph F. Payack
}

enriquevvazquez@merck.com

Supporting Information. Reactions were carried out under an atmosphere of dry nitrogen. Reagents and solvents were used as received from commercial sources. LDA was obtained from commercial sources as a 2.0 M solution in $\mathrm{n}$ heptane/ethylbenzene/THF. Triisopropylborate was reagent grade. THF contained $<200$ $\cdot \mathrm{g} / \mathrm{mL}$ water as determined by Karl-Fischer titration. All reactions were monitored by HPLC analysis using a Zorbax SB-C18, 4.6 x $250 \mathrm{~mm}$ column. Eluent: $\mathrm{CH}_{3} \mathrm{CN}$ and a 0.1 $\% \mathrm{H}_{3} \mathrm{PO}_{4} / 20 \mathrm{mM} \mathrm{NaClO}_{4}$ buffer. Initial conditions $70 \% \mathrm{CH}_{3} \mathrm{CN}$, with a gradient to $90 \%$ $\mathrm{CH}_{3} \mathrm{CN}$ over $7 \mathrm{~min}$. Flow rate: $1.5 \mathrm{~mL} / \mathrm{min}$, UV detection at $220 \mathrm{~nm}$.

General procedure for the preparation of N-Boc indoles. To a solution of 5-cyano indole $(5.00 \mathrm{~g} ; 35.2 \mathrm{mmol})$ and DMAP $(0.086 \mathrm{~g} ; 0.704 \mathrm{mmol})$ in $\mathrm{CH}_{2} \mathrm{Cl}_{2}(25 \mathrm{ml})$ was added Boc anhydride $(8.45 \mathrm{~g} ; 38.7 \mathrm{mmol})$. Stirred at room temperature. After $45 \mathrm{~min}$ the reaction was quenched with $1 \mathrm{~N} \mathrm{HCl}(25 \mathrm{ml})$. The organic layer was separated and dried over $\mathrm{MgSO}_{4}$. Filtered and concentrated to yield a solid. The solid was dissolved in hot 
methanol $\left(60^{\circ} \mathrm{C}\right)$ then cooled down to room temperature. Water $(100 \mathrm{ml})$ was added dropwise to crystallized product. Filtered and dried to give $1 \mathrm{~g}$ (8.25gm, 96.7\% yield).

General procedure for the preparation of 2-indolylborates. To a solution of $N$-Boc4-chloroindole 1a (2.00 g; $7.95 \mathrm{mmol})$ in THF (10 mL) was added triisopropylborate ( 2.8 $\mathrm{mL} ; 12.1 \mathrm{mmol})$. The solution was cooled to $0-5{ }^{\circ} \mathrm{C}$ in an ice bath and LDA (2.0 M, 10 mmol) was added over $1 \mathrm{hr}$. After $30 \mathrm{~min}$. the reaction was quenched by the addition of $2 \mathrm{~N} \mathrm{HCl}$. The organic layer was separated, dried over $\mathrm{MgSO}_{4}$ and concentrated to a solid which was recrystallized from acetonitrile/water to give 2a (2.34 g, 99\% yield); m.p. 97.8-99.0 ${ }^{\circ} \mathrm{C} ;{ }^{1} \mathrm{H}$ NMR (DMSO-d $\left.{ }^{6}, 400 \mathrm{MHz}\right) \delta 8.40$ (2H, br.s), $8.06-8.01(1 \mathrm{H}, \mathrm{m}), 7.29$ $-7.24(2 \mathrm{H}, \mathrm{m}), 6.63(1 \mathrm{H}, \mathrm{s}), 1.59(9 \mathrm{H}, \mathrm{s}) ;{ }^{13} \mathrm{C}$ NMR (DMSO-d $\left.{ }^{6}, 100 \mathrm{MHz}\right) \delta 150.0,140.6$ (br.) 137.4, 129.3, 125.5, 125.0, 122.6, 114.0, 109.7, 85.2, 28.0; Anal. Calcd for $\mathrm{C}_{13} \mathrm{H}_{15} \mathrm{BClNO}_{4}: \mathrm{C}, 52.83 ; \mathrm{H}, 5.12 ; \mathrm{N}, 4.74 ; \mathrm{Cl}, 12.0$. Found: C, 52.85; H, 4.99; N, 4.66; Cl, 11.86 .

2b $2.22 \mathrm{~g}\left(94 \%\right.$ yield); m.p. $98.2-99.9^{\circ} \mathrm{C}$; ${ }^{1} \mathrm{H}$ NMR (DMSO-d $\left.{ }^{6}, 400 \mathrm{MHz}\right) \delta 8.27(2 \mathrm{H}$, s), $8.09(1 \mathrm{H}, \mathrm{d}, J=8.8 \mathrm{~Hz}), 7.64(1 \mathrm{H}, \mathrm{d}, J=1.7 \mathrm{~Hz}), 7.30(1 \mathrm{H}, \mathrm{d}, J=8.8,1.9 \mathrm{~Hz}), 6.63$ $(1 \mathrm{H}, \mathrm{s}), 1.61(9 \mathrm{H}, \mathrm{s}) ;{ }^{13} \mathrm{C}$ NMR (DMSO-d 6 , $\left.100 \mathrm{MHz}\right) \delta 150.1,140.8,135.1,132.6$, 127.4, 124.2, 120.4, 116.3, 111.7, 85.0, 28.0; Anal. Calcd for $\mathrm{C}_{13} \mathrm{H}_{15} \mathrm{BClNO}_{4}: \mathrm{C}, 52.83 ; \mathrm{H}$, 5.12; N, 4.74; Cl,12.00. Found: C, 52.84; H, 4.65; N, 4.74; Cl, 11.95.

2c 2.31 g (96\%yield); m.p. $104-106{ }^{\circ} \mathrm{C}$; ${ }^{1} \mathrm{H}$ NMR (DMSO-d $\left.{ }^{6}, 400 \mathrm{MHz}\right) \delta 8.19$ (2H, s), $8.10(1 \mathrm{H}, \mathrm{d}, J=8.24 \mathrm{~Hz}), 7.57(1 \mathrm{H}, \mathrm{d}, J=7.60 \mathrm{~Hz}), 7.28(1 \mathrm{H}, \mathrm{ddd}, J=8.1,7.2,1.0 \mathrm{~Hz})$ $7.20(1 \mathrm{H}, \mathrm{t}, J=7.3 \mathrm{~Hz}), 6.63(1 \mathrm{H}, \mathrm{s}), 1.61(9 \mathrm{H}, \mathrm{s}) ;{ }^{13} \mathrm{C}$ NMR (DMSO-d $\left.{ }^{6}, 100 \mathrm{MHz}\right) \delta$ 
150.4, 138.5, 136.6, 131.1, 124.4, 123.0, 121.1, 115.0, 112.6, 84.4, 28.1; Anal. Calcd for $\mathrm{C}_{13} \mathrm{H}_{16} \mathrm{BNO}_{4}: \mathrm{C}, 59.80 ; \mathrm{H}, 6.18 ; \mathrm{N}, 5.36$. Found: $\mathrm{C}, 59.84 ; \mathrm{H}, 6.26 ; \mathrm{N}, 5.29$.

2d 2.12g (92\% yield); m.p. 97.7-99.3 ${ }^{\circ} \mathrm{C} .{ }^{1} \mathrm{H}$ NMR (DMSO-d $\left.{ }^{6}, 400 \mathrm{MHz}\right) \delta 8.26(2 \mathrm{H}$, s), $8.02(1 \mathrm{H}, \mathrm{d}, J=8.8 \mathrm{~Hz}), 7.77(1 \mathrm{H}, \mathrm{s}), 7.41(1 \mathrm{H}, \mathrm{d}, J=8.8 \mathrm{~Hz}), 6.62(1 \mathrm{H}, \mathrm{s}), 1.60(9 \mathrm{H}$, s); ${ }^{13} \mathrm{C}$ NMR (DMSO-d $\left.{ }^{6}, 100 \mathrm{MHz}\right) \delta 150.1,140.6,135.5,133.2,128.7,126.8,123.5$, 116.7, 115.5, 111.6, 85.0, 28.0; Anal. Calcd for $\mathrm{C}_{13} \mathrm{H}_{15} \mathrm{BBrNO}_{4}$ : C, 45.93; H, 4.45; N, 4.12; Br, 23.50. Found: C, 45.99; H, 4.38; N, 3.91; Br, 23.37

2e 2.11 g (89\% yield); m.p. $110-112{ }^{\circ} \mathrm{C} .{ }^{1} \mathrm{H}$ NMR (DMSO-d $\left.{ }^{6}, 400 \mathrm{MHz}\right) \delta 8.17(2 \mathrm{H}, \mathrm{s})$, $7.97(1 \mathrm{H}, \mathrm{d}, J=8.9 \mathrm{~Hz}), 7.09(1 \mathrm{H}, \mathrm{s}), 6.89(1 \mathrm{H}, \mathrm{d}, J=8.9 \mathrm{~Hz}), 6.57(1 \mathrm{H}, \mathrm{s}), 3.78(3 \mathrm{H}, \mathrm{s})$, $1.60(9 \mathrm{H}, \mathrm{s}) ;{ }^{13} \mathrm{C}$ NMR (DMSO-d $\left.{ }^{6}, 100 \mathrm{MHz}\right) \delta 155.9,150.4,139.7,132.1,131.3,115.6$, 113.0, 112.6, 103.6, 84.2, 55.8, 28.1; Anal. Calcd for $\mathrm{C}_{14} \mathrm{H}_{18} \mathrm{BNO}_{5}$ : C, 57.76; H, 6.23; N, 4.81. Found: C, 58.03; H, 6.24; N, 4.66.

2f $2.03 \mathrm{~g}$ (85\% yield); m.p. $110-112{ }^{\circ} \mathrm{C}$; ${ }^{1} \mathrm{H}$ NMR (DMSO-d $\left.{ }^{6}, 400 \mathrm{MHz}\right) \delta 8.16(2 \mathrm{H}, \mathrm{s})$, $7.97(1 \mathrm{H}, \mathrm{d}, J=8.1 \mathrm{~Hz}), 7.35(1 \mathrm{H}, \mathrm{s}), 7.09(1 \mathrm{H}, \mathrm{d}, J=8.2 \mathrm{~Hz}), 6.56(1 \mathrm{H}, \mathrm{s}), 2.38(3 \mathrm{H}, \mathrm{s})$, $1.61(9 \mathrm{H}, \mathrm{s}) ;{ }^{13} \mathrm{C}$ NMR (DMSO-d $\left.{ }^{6}, 100 \mathrm{MHz}\right) \delta 150.4,139.0,135.0,131.8,131.4,125.7$, 120.9, 114.7, 112.5, 84.2, 28.1, 21.4; Anal. Calcd for $\mathrm{C}_{13} \mathrm{H}_{18} \mathrm{BNO}_{4}$ : C, 61.12; H, 6.59; N, 5.09. Found: C, 61.27; H, 6.72; N, 4.76.

2 g 1.91 g (81\% yield); m.p. $245-248{ }^{\circ} \mathrm{C} ;{ }^{1} \mathrm{H}$ NMR (DMSO-d $\left.{ }^{6}, 400 \mathrm{MHz}\right) \delta 8.29(2 \mathrm{H}, \mathrm{s})$, $8.18(1 \mathrm{H}, \mathrm{d}, J=8.6 \mathrm{~Hz}), 8.08(1 \mathrm{H}, \mathrm{d}, J=1.1 \mathrm{~Hz}), 7.64(1 \mathrm{H}, \mathrm{dd}, J=8.7,1.4 \mathrm{~Hz}), 6.70$ $(1 \mathrm{H}, \mathrm{s}), 1.57(9 \mathrm{H}, \mathrm{s}) ;{ }^{13} \mathrm{C}$ NMR (DMSO-d 6 , $\left.100 \mathrm{MHz}\right) \delta 149.9,141.9,138.5,131.3,127.5$, 126.1, 120.2, 115.9, 112.0, 105.4, 85.7, 28.0; Anal. Calcd for $\mathrm{C}_{14} \mathrm{H}_{15} \mathrm{BN}_{2} \mathrm{O}_{4}: \mathrm{C}, 58.77 ; \mathrm{H}$, 5.28; N, 9.79. Found: C, 58.72; H, 5.25; N, 9.74. 
2h 1.74 g (73\% yield); m.p. $231-234{ }^{\circ} \mathrm{C} ;{ }^{1} \mathrm{H}$ NMR (DMSO-d 6 , $\left.400 \mathrm{MHz}\right) \delta 8.20(2 \mathrm{H}$, s), $8.11(1 \mathrm{H}, \mathrm{dd}, J=4.7,4.3 \mathrm{~Hz}), 7.32(1 \mathrm{H}, \mathrm{dd}, J=6.8,2.3 \mathrm{~Hz}), 7.08(1 \mathrm{H}, \mathrm{ddd}, J=9.2$, 9.2, 2.4Hz), $6.63(1 \mathrm{H}, \mathrm{s}), 1.61(9 \mathrm{H}, \mathrm{s}) ;{ }^{13} \mathrm{C}$ NMR (DMSO-d $\left.{ }^{6}, 100 \mathrm{MHz}\right) \delta 158.4(\mathrm{~d}, J=$ $236.3 \mathrm{~Hz}), 149.6,140.6,132.6,131.6(\mathrm{~d}, J=9.9 \mathrm{~Hz}), 115.5(\mathrm{~d}, J=9.4 \mathrm{~Hz}), 111.6(\mathrm{~d}, J=$ 3.6 Hz), $111.3(\mathrm{~d}, J=25.1 \mathrm{~Hz}), 105.8(\mathrm{~d}, J=23.4 \mathrm{~Hz}), 84.2,27.5 ;{ }^{19} \mathrm{~F}$ NMR (DMSO-d ${ }^{6}$, $376 \mathrm{MHz}) \delta$-121.6; Anal. Calcd for $\mathrm{C}_{13} \mathrm{H}_{15} \mathrm{BFNO}_{4}$ : C, 55.95; H, 5.42; N, 5.02; F, 6.81 . Found: C, 56.23; H, 5.42; N, 4.72; F, 5.62.

General Procedure for silanes or siloxanes. To a solution of $N$-Boc-5-chloroindole 1 b $(2.00 \mathrm{~g}, 7.95 \mathrm{mmol})$ in THF $(10 \mathrm{~mL})$ was added chlorotrimethylsilane $(1.5 \mathrm{~mL}$, $11.8 \mathrm{mmol})$. The solution was cooled to $0^{\circ} \mathrm{C}$ in an ice bath. LDA (2.0 $\mathrm{M}$ in THF, 10 mmol) was added over $1 \mathrm{hr}$. After the addition was complete the reaction was aged for 30 minutes and quenched with water $(10 \mathrm{~mL})$. The organic layer was separated and dried over $\mathrm{MgSO}_{4}$. The organics are filtered and concentrated. Flash chromatography on silica gel (5\% EtOAc /hexane) gave 3a as a colorless solid (2.15 g, 84\% yield); m.p. 95.2$96.3^{\circ} \mathrm{C} ;{ }^{1} \mathrm{H}$ NMR (DMSO-d $\left.{ }^{6}, 400 \mathrm{MHz}\right) \delta 7.86(1 \mathrm{H}, \mathrm{d}, J=9 \mathrm{~Hz}), 7.62(1 \mathrm{H}, \mathrm{d}, J=2 \mathrm{~Hz})$, $7.28(1 \mathrm{H}, \mathrm{dd}, J=9,2 \mathrm{~Hz}), 6.84(1 \mathrm{H}, \mathrm{s}), 1.62(9 \mathrm{H}, \mathrm{s}), 0.26(9 \mathrm{H}, \mathrm{s}) ;{ }^{13} \mathrm{C}$ NMR (DMSO-d ${ }^{6}$, $100 \mathrm{MHz}) \delta 150.9,143.5,136.1,132.4,127.5,124.9,120.6,119.1,117.0,85.3,28.1$, 0.4; Anal. Calcd for $\mathrm{C}_{16} \mathrm{H}_{22} \mathrm{ClNO}_{2} \mathrm{Si}$ : C, 59.33, H, 6.85, N, 4.32, Cl,10.95, Si, 8.67. Found: C, 59.79, H, 6.82, N, 4.12, Si, 8.69.

3b $1.95 \mathrm{~g}$ (78\% yield); ${ }^{1} \mathrm{H}$ NMR (DMSO-d $\left.{ }^{6}, 400 \mathrm{MHz}\right) \delta 7.86(1 \mathrm{H}, \mathrm{d}, J=8.5 \mathrm{~Hz}), 7.37$ $(1 \mathrm{H}, \mathrm{s}), 7.14(1 \mathrm{H}, \mathrm{d}, J=8.6 \mathrm{~Hz}), 6.88(1 \mathrm{H}, \mathrm{s}), 4.53(1 \mathrm{H}, \mathrm{m}, J=3.4 \mathrm{~Hz}), 2.37(3 \mathrm{H}, \mathrm{s}), 1.65$ $(9 \mathrm{H}, \mathrm{s}), 0.35(6 \mathrm{H}, \mathrm{d}, J=3.4 \mathrm{~Hz}) ;{ }^{13} \mathrm{C} \mathrm{NMR}\left(\mathrm{DMSO}^{-\mathrm{d}}{ }^{6}, 100 \mathrm{MHz}\right) \delta 151.3,138.7,136.1$, 
132.1, 131.2, 126.8, 121.1, 120.6, 115.3, 85.0, 28.2, 21.3, -2.3; Anal. Calcd for $\mathrm{C}_{16} \mathrm{H}_{23} \mathrm{NO}_{2} \mathrm{Si}: \mathrm{C}, 66.39, \mathrm{H}, 8.01, \mathrm{~N}, 4.84, \mathrm{Si}$, 9.70. Found: C, 66.31, H, 8.18, N, 4.52, Si, 9.61.

3c The reaction was quenched with a saturated aqueous solution of sodium bicarbonate $(10 \mathrm{~mL})$ and stirred for one hour. The organic layer was dried over magnesium sulfate and concentrated to a semi-solid. 6.0g (91\% yield); ${ }^{1} \mathrm{H}$ NMR (DMSO-d $\left.{ }^{6}, 400 \mathrm{MHz}\right) \delta 7.81$ $(1 \mathrm{H}, \mathrm{d}, J=8.5 \mathrm{~Hz}), 7.38(1 \mathrm{H}, \mathrm{s}), 7.11(1 \mathrm{H}, \mathrm{d}, J=8.4 \mathrm{~Hz}), 6.94(1 \mathrm{H}, \mathrm{s}), 5.67(1 \mathrm{H}, \mathrm{s}), 2.37$ $(3 \mathrm{H}, \mathrm{s}), 1.65(9 \mathrm{H}, \mathrm{s}), 0.32(6 \mathrm{H}, \mathrm{s}) ;{ }^{13} \mathrm{C}$ NMR (DMSO-d $\left.{ }^{6}, 125 \mathrm{MHz}\right) \delta 150.8,141.7,135.2$, $131.3,130.8,125.9,120.6,119.3,114.8,84.1,27.6,20.7,1.4 ;{ }^{29} \mathrm{Si}$ NMR (DMSO-d ${ }^{6}, 99.3$ MHz) $\delta$-5.9; Anal. Calcd for $\mathrm{C}_{16} \mathrm{H}_{23} \mathrm{NO}_{3} \mathrm{Si}$ : C,62.92, H, 7.59, N, 4.59, Si, 9.19. Found: C, 62.46, H, 7.66, N, 4.33, Si, 9.01 . 